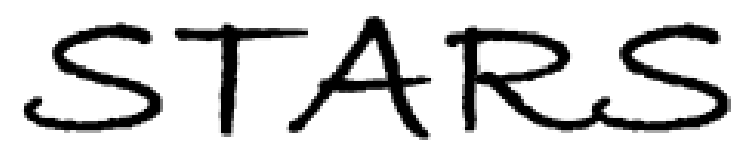

University of Central Florida

STARS

Faculty Bibliography 2000s

Faculty Bibliography

$1-1-2008$

\title{
Stochastic sensing of relative anisotropic polarizabilities
}

\author{
S. Sukhov \\ University of Central Florida \\ D. Haefner \\ University of Central Florida
}

A. Dogariu

University of Central Florida

Find similar works at: https://stars.library.ucf.edu/facultybib2000

University of Central Florida Libraries http://library.ucf.edu

This Article is brought to you for free and open access by the Faculty Bibliography at STARS. It has been accepted for inclusion in Faculty Bibliography 2000s by an authorized administrator of STARS. For more information, please contactSTARS@ucf.edu.

\section{Recommended Citation}

Sukhov, S.; Haefner, D.; and Dogariu, A., "Stochastic sensing of relative anisotropic polarizabilities" (2008). Faculty Bibliography 2000s. 1024.

https://stars.library.ucf.edu/facultybib2000/1024

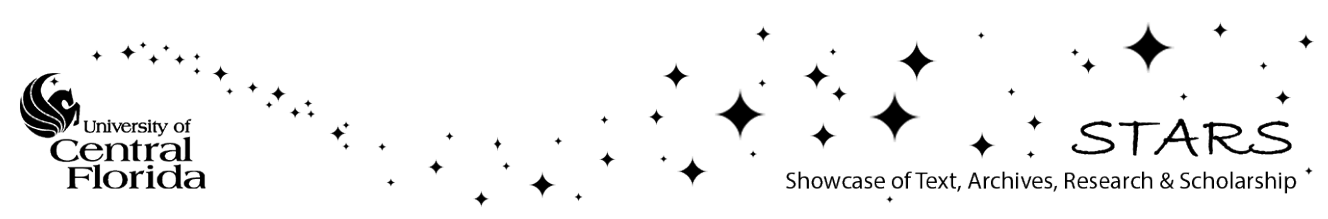




\title{
Stochastic sensing of relative anisotropic polarizabilities
}

\author{
S. Sukhov, D. Haefner, and A. Dogariu \\ CREOL, College of Optics and Photonics, University of Central Florida, Orlando, Florida 32816-2700, USA
}

(Received 5 December 2007; published 16 April 2008)

\begin{abstract}
We describe the concept of stochastic scattering polarimetry. This method allows determination of the anisotropic polarizability of a scattering object using a statistical analysis of the polarimetrically measured intensity distributions in the wave zone (far field). We show that this anisotropic polarizability may be determined even in situations where the state of polarization of the incident field is not known. The efficiency of the recovering procedure is demonstrated by several examples of light scattering in both far- and near-field geometries.
\end{abstract}

DOI: 10.1103/PhysRevA.77.043820

PACS number(s): 42.25.Fx, 42.25.Ja, 78.67.-n

\section{INTRODUCTION}

Recently, there has been a great deal of interest in characterizing the alignment or shape of nanoparticles or single molecules [1,2]. Understanding the shape of nanoparticles can lead to better fabrication and design procedures, and may help in characterizing properties of inorganic nanostructures or small biological objects. Among various methods, optical probing offers some unique advantages; for instance, due to the sensitive nature of biological media, it is often required to perform this characterization noninvasively using optical fields. However, in many situations, the characterization procedure is complicated by the random nature of the particle's orientation. Quantitative information may still be obtained using the concepts of statistical optics when experimental scattering data are processed as distributions rather than images.

Many sensing processes can be described in terms of a scattering phenomena, where the outcome relates to both the excitation field and the material properties under scrutiny. In a typical scattering experiment, the object is probed with a controlled incident field for which the Stokes vector is known. Using combinations of different polarizations of the incident field and polarimetric measurements, the unknown polarizability may be calculated. Equivalently, the polarizability can be found when keeping the initial polarization constant and rotating through all orientations of source and detector configurations.

A second possibility for obtaining information about an unknown polarizability is a situation where the incident field is unknown and the observation point is fixed. In this case, an ensemble of intensities is accumulated for different orientations of the effective polarizability. This approach is preferable when objects rotate in time, for example, in colloidal solutions, or in the case of near-field microscopy where the local polarizability acquires different orientations during the scan. Without direct control of the incident field, the material properties cannot be determined from the scattered intensity alone. However, if one examines the intensity fluctuations, the information can be extracted. We have recently introduced the method of the stochastic polarimetry which uses the statistical properties of the ensemble of scattered intensities for retrieving the anisotropic polarizability of a scatterer [3]. As we will show in the following, in the practice of stochastic scattering polarimetry one is not required to know or control the exact polarization of the incident field; the only constraint is that the incident field be constant in direction, irradiance, and polarization properties for the different orientations of the scatterer.

\section{STOCHASTIC POLARIMETRY}

A generic scattering experiment, dealing with a real polarizability (absorption-free) and a fully polarized excitation, is commonly described in terms of the cross-spectral density matrix [4] as follows:

$$
W_{o b s}=\left[\begin{array}{cc}
E_{x} E_{x}^{*} & E_{x} E_{y}^{*} \\
E_{y} E_{x}^{*} & E_{y} E_{y}^{*}
\end{array}\right]=P^{t} R_{1} \alpha R_{1}^{t} R_{2} W_{i n c} R_{2}^{t} R_{1} \alpha R_{1}^{t} P,
$$

where $W_{o b s}$ and $W_{\text {inc }}$ are the cross-spectral density matrices of the scattered and excitation fields. In Eq. (1), the $x y$ plane is chosen to be perpendicular to the direction of propagation (Fig. 1), and $P$ is a $3 \times 2$ tensor that represents the propagation to the observation point in the wave zone. In Eq. (1), $R_{1}$ and $R_{2}$ account for the three-dimensional rotation of the diagonalized polarizability and of the excitation polarization into the detection coordinate frame, while $\alpha$ denotes a diagonalized form of the polarizability as follows:

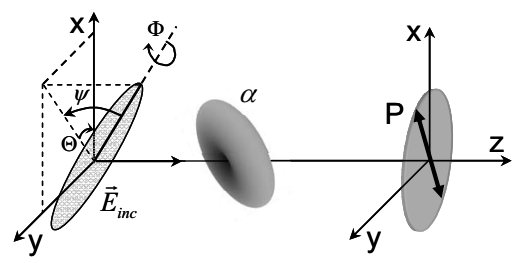

FIG. 1. A generic scattering process where a scatterer with unknown polarizabity $\alpha$ is illuminated by a constant, arbitrarily polarized field $E_{\text {inc }}$. An intensity measurement is performed in the far field through a polarizer $P$ oriented in the plane $x y . \Theta$ is the angle between the projection of the major polarization axis onto the $x y$ plane and the $x$ axis, $\psi$ is the angle between the major polarization axis and the $x y$ plane, and angle $\Phi$ specifies the rotation of the polarization ellipse around the major axis. 


$$
\alpha=\left[\begin{array}{lll}
a & 0 & 0 \\
0 & b & 0 \\
0 & 0 & c
\end{array}\right] .
$$

The expression of the cross-spectral density in Eq. (1) contains both the correlation between orthogonal field components and the intensities along two orthogonal directions $x$ and $y$. In a polarimetric scattering experiment, one measures only intensities $I_{i}=W_{i i}^{o b s}(i=x, y)$; for example, by the use of two polarizers, the diagonal elements of cross-spectral density can be determined. For a given incident field described by $W_{i n c}$, the scattered intensities are obtained for different orientations of the scatterer. An ensemble of scattered intensities can be further analyzed statistically to infer the information about polarizability $\alpha$. In the case of isotropic substances the morphological information included in the polarizability can be also retrieved. In our case, the quantitative analysis will be based on evaluating statistical moments of the form

$$
M_{i_{1} i_{2}, \ldots, i_{n}}^{(n)}=\int_{\Omega} I_{i_{1}}(\Omega) I_{i_{2}}(\Omega), \ldots, I_{i_{n}}(\Omega) p(\Omega) d \Omega,
$$

where $i_{1}, i_{2}, \ldots, i_{n}=x, y$ and $p(\Omega)$ represents the probability function associated with the orientation of the diagonalized polarizability. The integration in Eq. (3) is performed over all orientations determined by solid angle $\Omega$.

Let us examine in more detail the situation where an anisotropic scatterer with an unknown polarizability $\alpha$ is illuminated by some constant arbitrarily polarized incident field as depicted in Fig. 1. In general, a fully polarized excitation field can be described in terms of three orientation angles and an ellipticity parameter; in other words, there are four independent parameters that determine the structure of the excitation field. The situation of interest here is one where during the experiment the scatterer rotates randomly in space as described by $p(\Omega)$. The scatterer interacts with the incident field and the scattered intensities are polarimetrically measured along two orthogonal directions $x$ and $y$ as shown in Fig. 1. For a given probability function describing the orientation confinement of the polarizability, expressions for the scattered intensity can be derived starting from Eq. (1). Furthermore, the moments of the intensity distributions can be related to the material properties and the parameters of the excitation field. All in all, the scattering situation is fully described by the three elements of the diagonalized polarizability tensor $\alpha$ [Eq. (2)] in addition to the four independent incident field parameters.

In performing polarimetric intensity measurements along two orthogonal directions $x$ and $y$, a situation arises when the incident field is polarized with the major axis oriented at the bisection of the two directions of the measurement. In this situation, all the moments of the two orthogonal intensity distributions are equal, and, consequently, the number of independent equations which can be used for reconstruction is reduced. Unique recovering of $\alpha$ may still be possible by considering higher-order moments of the intensity distributions to obtain additional independent relationships. However, in this case one has to recognize that higher-order mo- ments of the intensity distributions are more susceptible to noise [5]. Alternatively, one can choose to consider only up to the second-order moments of intensity distributions, but introduce an additional intensity measurement. For example, by performing a third measurement along the bisection of the first two orthogonal measurements, a rotation of the detection coordinate system can be applied which decreases the cross correlation of orthogonal intensity distributions. The parameters characterizing the incident field and the material properties are uncorrelated; as such, decreasing the cross correlation of intensity ensembles allows for more independent probability distribution functions. Note that the rotation of the detection coordinate system is a linear transformation performed over all the elements of the intensity distributions, and, therefore, no statistical information is lost. This rotation angle $\Theta$ of the coordinate system, $\widetilde{W}_{\text {obs }}=R(\Theta) W_{\text {obs }} R(\Theta)^{-1}$, can be found by minimizing the real part of the cross correlation between the two orthogonal measurements, which is related to the intensity polarized at their bisection angle.

Let us consider the effect of the rotation

$$
R(\Theta)=\left(\begin{array}{cc}
\cos \Theta & -\sin \Theta \\
\sin \Theta & \cos \Theta
\end{array}\right)
$$

on the first moment of cross-spectral density matrix as follows:

$$
\left\langle W_{o b s}\right\rangle=\left[\begin{array}{cc}
\left\langle I_{x}\right\rangle & \left\langle E_{x}^{*} E_{y}\right\rangle \\
\left\langle E_{y}^{*} E_{x}\right\rangle & \left\langle I_{y}\right\rangle
\end{array}\right]=\left[\begin{array}{cc}
M_{x}^{(1)} & \left\langle E_{x}^{*} E_{y}\right\rangle \\
\left\langle E_{y}^{*} E_{x}\right\rangle & M_{y}^{(1)}
\end{array}\right] .
$$

Here the angle brackets denote ensemble average. Minimizing the real part of the off-diagonal element the first ordermoment $\left\langle\widetilde{W}_{\text {obs }}\right\rangle=R(\Theta)\left\langle W_{\text {obs }}\right\rangle R(\Theta)^{-1}$ leads to the following condition:

$$
\operatorname{Re}\left\langle E_{x}^{*} E_{y}\right\rangle\left(\cos ^{2} \Theta-\sin ^{2} \Theta\right)+\left(\left\langle I_{x}\right\rangle-\left\langle I_{y}\right\rangle\right) \cos \Theta \sin \Theta=0 .
$$

In fact, $\Theta$ defines the angle between the $x$ axis and the projection of the major axis of the polarization ellipse of the incident field onto the $x y$ plane (Fig. 1). The rotation we have identified eliminates the dependence on this angle and, therefore, in the new system of coordinates, the incident field is now described by only three independent parameters.

The third additional measurement at $45^{\circ}$ with respect to the first two orthogonal directions can be expressed in terms of the off-diagonal elements of the cross-correlation matrix as follows:

$$
M_{45}^{(1)}=\frac{1}{2} M_{x}^{(1)}+\frac{1}{2} M_{y}^{(1)}+\frac{1}{2}\left\langle E_{x}^{*} E_{y}\right\rangle+\frac{1}{2}\left\langle E_{x} E_{y}^{*}\right\rangle,
$$

where $M_{45}^{(1)}$ is the first moment of the intensity distribution measured at $45^{\circ}$. Using Eqs. (6) and (7), the angle $\Theta$ is finally evaluated to be 


$$
\Theta=\tan ^{-1}\left(\frac{M_{x}^{(1)}-M_{y}^{(1)} \pm \sqrt{2\left(M_{x}^{(1)}\right)^{2}+2\left(M_{y}^{(1)}\right)^{2}+4 M_{45}^{(1)}\left(M_{45}^{(1)}-M_{x}^{(1)}-M_{y}^{(1)}\right)}}{2 M_{45}^{(1)}-M_{x}^{(1)}-M_{y}^{(1)}}\right) .
$$

In Eq. (8), the sign is chosen such that in the new coordinate system the condition $M_{x x}^{(1)}>M_{y y}^{(1)}$ is fulfilled.

In the rotated system of coordinates, two new intensity distributions $\tilde{I}_{x}=\tilde{W}_{x x}^{o b s}$ and $\tilde{I}_{y}=\tilde{W}_{y y}^{o b s}$ can be defined as

$$
\begin{aligned}
& \tilde{I}_{x}=I_{x} \cos ^{2} \Theta+I_{y} \sin ^{2} \Theta-2\left(I_{45}-\frac{1}{2} I_{x}-\frac{1}{2} I_{y}\right) \cos \Theta \sin \Theta, \\
& \tilde{I}_{y}=I_{x} \sin ^{2} \Theta+I_{y} \cos ^{2} \Theta+2\left(I_{45}-\frac{1}{2} I_{x}-\frac{1}{2} I_{y}\right) \cos \Theta \sin \Theta,
\end{aligned}
$$

where $I_{45}$ is the intensity measured along the $45^{\circ}$ direction.

The first and second moments of distributions $\tilde{W}_{x x}^{o b s}$ and $\tilde{W}_{y y}^{o b s}$ can now be evaluated together with the corresponding cross-correlation term $M_{x y}^{(2)}$. Using Eqs. (1)-(3) and assuming that $p(\Omega)$ corresponds to a uniform random distribution of the orientation of the diagonalized polarizability, one finds the first moments of the two rotated ensembles to be

$$
\begin{gathered}
M_{x}^{(1)}=\frac{1}{5}\left[\left(Q_{1}+3\right) \varepsilon+3+K\right] \delta_{1}+Q_{1} \varepsilon \bar{\alpha}^{2}+\bar{\alpha}^{2} K, \\
M_{y}^{(1)}=\frac{1}{5}[(L+3) \varepsilon+3] \delta_{1}+\bar{\alpha}^{2} \varepsilon L, \\
M_{i j}^{(2)}=P_{i j}^{(1)}(\varepsilon) \delta_{1}^{2}+P_{i j}^{(2)}(\varepsilon) \delta_{1} \bar{\alpha}^{2}+P_{i j}^{(3)}(\varepsilon) \delta_{2} \bar{\alpha}+P_{i j}^{(4)}(\varepsilon) \bar{\alpha}^{4} .
\end{gathered}
$$

In the general expressions for the second-order moments in Eq. $(10), P_{i j}^{(n)}(\varepsilon)$ are second-order polynomials with respect to the ellipticity parameter $\varepsilon$. The exact expressions for these polynomials are given in the Appendix. In addition, the following shorthand notations were used:

$$
\begin{gathered}
\delta_{1}=\left(d b^{2}+d b d c+d c^{2}\right) / 3, \quad \delta_{2}=d b d c(d b+d c) / 2, \\
d b=b-\bar{\alpha}, \quad d c=c-\bar{\alpha}, \quad \bar{\alpha}=(a+b+c) / 3, \\
L=\cos ^{2} \Phi, \quad K=\cos ^{2} \psi, \quad Q_{1}=\sin ^{2} \Phi \sin ^{2} \psi, \quad \varepsilon=(w / \ell)^{2},
\end{gathered}
$$

where $\ell$ and $w$ are the main axes of the excitation polarization ellipse $(\ell>w)$; the meaning of the angles $\Phi$ and $\psi$ is explained in Fig. 1.

The expressions in Eq. (10) constitute the main result of this analysis, as they establish the relationship between the statistical moments (up to the second order) of the measured intensities and the parameters defining the anisotropic polarizability to be determined. It is worth mentioning that the values $a, b$, and $c$ used in Eq. (11) refer to relative values of the polarizability tensor, rather than the actual values of the diagonalized tensor elements; also, a constant has been fac- tored out and included in the propagation operator $P$. These relative values of the diagonal elements allow generating a number of validity criteria for our reconstruction procedure. Using the dimensionless variables $\delta_{1} / \bar{\alpha}^{2}, \delta_{2} / \bar{\alpha}^{3}, K, L$, and $\varepsilon$ in Eq. (10), one finds that they are subject to the following physical restrictions:

$$
\delta_{1} / \bar{\alpha}^{2} \in[0,1], \quad \delta_{2} / \bar{\alpha}^{3} \in[-1,1 / 8]
$$

$$
\varepsilon(1-K+K L) \leq K \leq 1, \quad L \in[0,1], \quad \varepsilon \in[0,1] .
$$

The restrictions on $K$ imply that the projection of the major axis of excitation polarization ellipse onto the $x y$ plane should be greater than the projection of its minor axis. Starting from the conditions expressed in Eq. (12) one can establish the following limiting values for the ratios between statistical moments:

$$
\begin{gathered}
M_{y}^{(1)} / M_{x}^{(1)} \in[0,1], \quad M_{x x}^{(2)} /\left(M_{x}^{(1)}\right)^{2} \in[1,3.1], \\
M_{y y}^{(2)} /\left(M_{x}^{(1)}\right)^{2} \in[0,2.1], \quad M_{x y}^{(2)} /\left(M_{x}^{(1)}\right)^{2} \in[0,1] .
\end{gathered}
$$

These relations may be useful in practice when, in the presence of experimental errors, the measured statistical moments may shift outside the validity region.

\section{EXAMPLES OF RECONSTRUCTION}

We have now established a direct relationship between five statistical moments of the polarimetric intensity measurements $\left(M_{x}^{(1)}, M_{y}^{(1)}, M_{x x}^{(2)}, M_{y y}^{(2)}\right.$, and $\left.M_{x y}^{(2)}\right)$ and the six unknown variables that determine the outcome of the random scattering process; three material parameters defining $\alpha$ and three parameters $(\Phi, \psi, \varepsilon)$ describing the incident field in the rotated system of coordinates. This situation represents still an underdetermined system of equations. However, any additional knowledge about the material properties or about the excitation field will allow a full description of the scattering situation, even if only the first- and second-order moments are being used.

Before illustrating several examples, it is worth mentioning that one can follow the procedure outlined above to obtain similar relations between the statistical moments of the measured intensities and material parameters for situations where the random orientation of the polarizability is described by other distribution functions. Also, the same treatment can be considered in circumstances where the experiment is performed using a polarimetric detection system based on the measurement of two circular polarizations and some elliptical state.

To test the theory outlined above, a series of numerical experiments were performed to model the interaction with a 
scatterer described by an anisotropic polarizability. The experiment was numerically simulated for random orientations of the scattering particle with respect to a fixed detection frame. For each orientation, the intensity was recorded in the three directions of polarization as described above.

\section{A. Far-field stochastic polarimetry}

One common assumption that can be made about excitation field properties is that the plane of the polarization ellipse of the excitation field is parallel to the detection plane (forward scattering). In this case, when $\psi=0$ and $\Phi=0$ (see Fig. 1) the expressions for the statistical moments in Eq. (10) become

$$
\begin{gathered}
M_{x}^{(1)}=\frac{1}{5}(3 \varepsilon+4) \delta_{1}+\bar{\alpha}^{2}, \\
M_{y}^{(1)}=\frac{1}{5}(4 \varepsilon+3) \delta_{1}+\bar{\alpha}^{2} \varepsilon, \\
M_{x x}^{(2)}=\frac{3}{35}\left(9 \varepsilon^{2}+8 \varepsilon+16\right) \delta_{1}^{2}+\frac{6}{5}(4+\varepsilon) \bar{\alpha}^{2} \delta_{1} \\
-\frac{8}{35}(8+3 \varepsilon) \delta_{2} \bar{\alpha}+\bar{\alpha}^{4}, \\
M_{y y}^{(2)}=\frac{3}{35}\left(16 \varepsilon^{2}+8 \varepsilon+9\right) \delta_{1}^{2}+\frac{6}{5}(4 \varepsilon+1) \varepsilon \bar{\alpha}^{2} \delta_{1} \\
-\frac{8}{35}(8 \varepsilon+3) \varepsilon \delta_{2} \bar{\alpha}+\varepsilon^{2} \bar{\alpha}^{4}, \\
M_{x y}^{(2)}=\frac{3}{35}\left(4 \varepsilon^{2}+17 \varepsilon+4\right) \delta_{1}^{2}+\frac{3}{5}\left(\varepsilon^{2}+1\right) \bar{\alpha}^{2} \delta_{1} \\
-\frac{4}{35}\left(3 \varepsilon^{2}-8 \varepsilon+3\right) \delta_{2} \bar{\alpha}+\varepsilon \bar{\alpha}^{4} .
\end{gathered}
$$

In practice, in order to determine the four unknowns $\bar{\alpha}, \delta_{1}, \delta_{2}, \varepsilon$ one needs only four of these equations. Because in an experiment the evaluation of the intensity moments is always subject to a certain procedural accuracy, we will choose a combination of the four equations that provides the minimal error in solving for the unknowns. Our estimations show that the evaluation of $M_{x}^{(1)}, M_{y}^{(1)}, M_{x x}^{(2)}$, and $M_{x y}^{(2)}$ is more stable with respect to possible experimental errors.

A series of simulations was performed for different elliptical excitation states. Table I summarizes the percent error of reconstruction for different shape parameters, different parameters of ellipticity, and different numbers of polarization measurements. The percent error in Table I refers to an average of the deviation in the calculation of the $b / a$ and $c / a$ values from the exact values of the polarizability. The polarizability of the particle is related to the shape parameters $\eta$, $\beta$, and $\gamma$ through the Clausius-Mossotti expression for isotropic ellipsoids [6] as follows:

$$
\alpha_{j}=4 \pi \eta \beta \gamma \frac{n^{2}-1}{3+3 L_{j}\left(n^{2}-1\right)},
$$

where $\alpha_{j}=a, b, c$, factors $L_{j}$ are determined by ellipsoid shape [6], and $n$ is the refractive index of the particle that was chosen to be equal to 1.5 for numerical computations.

Obviously, the reconstruction error decreases with increasing the number of realizations of particle orientation. Also, one can notice that the error increases for larger values of ellipticity parameter $\varepsilon$, and this can be understood by considering the limiting case of circularly polarized excitation. In this situation, all the moments for the linear polarization measurements in the $x$ and $y$ directions become equal, and the system of equations becomes undetermined. In the case of circularly polarized excitation, the morphological properties of the material may be found based on circular polarization measurements, however, this situation will not be discussed here. Another detail which we would like to point out is that according to our numerical results the reconstruction error seems to be smaller for purely anisotropic polarizabilities when $a \neq b \neq c$.

Another practical situation that is often of interest is when the incident field is in some arbitrary linear state of polarization. In this case, the general expression in Eq. (10) reduces to the following system of equations:

$$
\begin{gathered}
M_{x}^{(1)}=\bar{\alpha}^{2} K+\frac{3+K}{5} \delta_{1}, \\
M_{y}^{(1)}=\frac{3}{5} \delta_{1}, \\
M_{x x}^{(2)}=\bar{\alpha}^{4} K^{2}+\frac{8 \bar{\alpha}(K-9) K}{35} \delta_{2}+\frac{42 \bar{\alpha}^{2} K(K+3)}{35} \delta_{1} \\
+\frac{3(3+K)^{2}}{35} \delta_{1}^{2}, \\
M_{x y}^{(2)}=-\frac{12 K \bar{\alpha}}{35} \delta_{2}+\frac{3 \bar{\alpha}^{2} K}{5} \delta_{1}+\frac{3(3+K)}{35} \delta_{1}^{2},
\end{gathered}
$$

which may be solved for the magnitudes of the diagonal elements $a, b, c$ of the polarizability tensor from Eq. (2), and the polar angle $\psi$. In this case, the choice of the optimal four equations is unequivocal because the linearly polarized incident field moments $M_{y}^{(1)}$ and $M_{y y}^{(2)}$ are no longer independent: $M_{y y}^{(2)}=(15 / 7)\left(M_{y}^{(1)}\right)^{2}$.

To test the method of stochastic scattering polarimetry in this situation, a series of numerical experiments was performed for various angles of the incident excitation, different anisotropies, and the different number of realizations of particle orientation. The results are displayed in Table II.

Examining the results in Table II, one can easily see that there is a strong dependence of the reconstruction error on the polar angle $\psi$. In the case when $\psi$ equals $\pm \pi / 2$, the electric field vector is directed along the path to the observation point, and the measured intensities no longer depend on orientation of the polarizer. In this situation, the equations for the $x$ and $y$ components of cross-spectral density matrix are 
TABLE I. Percentage error of reconstructed polarizability for different shapes of particles, different field orientations, and different numbers of realizations for the case of an elliptically polarized excitation field, $\psi=0, \Phi=0$.

\begin{tabular}{cccccc}
\hline \hline & Shape parameters & \multicolumn{4}{c}{ Realizations } \\
$\varepsilon$ & $\eta, \beta, \gamma$ & 500 & 1000 & 2500 & 5000 \\
\hline \multirow{3}{*}{0} & $3,3,1$ & 2.0 & 2.0 & 1.8 & 1.1 \\
& $3,2,1$ & 1.0 & 0.7 & 0.5 & 0.3 \\
& $3,1,1$ & 2.3 & 1.3 & 1.7 & 0.8 \\
0.15 & $3,3,1$ & 2.0 & 1.4 & 1.4 & 2.0 \\
& $3,2,1$ & 1.0 & 0.7 & 0.6 & 0.3 \\
& $3,1,1$ & 1.4 & 1.9 & 1.0 & 0.6 \\
0.3 & $3,3,1$ & 3.5 & 2.3 & 2.4 & 2.4 \\
& $3,2,1$ & 2.5 & 1.5 & 1.0 & 1.0 \\
& $3,1,1$ & 3.2 & 2.7 & 2.4 & 1.7 \\
\multirow{3}{*}{0.65} & $3,3,1$ & 5.6 & 5.4 & 3.3 & 2.4 \\
& $3,2,1$ & 4.3 & 3.7 & 2.3 & 1.3 \\
& $3,1,1$ & 5.5 & 4.5 & 2.8 & 3.2 \\
\hline \hline
\end{tabular}

no longer independent. However, it is remarkable that in the case when the polar angle $\psi$ is less than $\pi / 3$, the original values of anisotropic polarizability can be recovered within about $2 \%$.

In order to illustrate the accuracy of the proposed method for reconstructing unknown polarizabilities, let us consider the simple example of scattering from a GaP nanorod (refractive index of 3.37), which is $100 \mathrm{~nm}$ long and has an aspect ratio of $1 / 10$. In this case, after 5000 intensity calculations for different orientations of nanorod, we are able to retrieve the ratio of polarizability tensor components $b / a$ $=0.20 \pm 0.05$, which is in very good agreement with the exact value $b / a=0.199$.

TABLE II. Percentage error of reconstructed polarizability for different shapes of particles, different field orientations, and different numbers of realizations for the case of a linearly polarized excitation field $(\varepsilon=0)$.

\begin{tabular}{cccccc}
\hline \hline & Shape parameters & \multicolumn{4}{c}{ Realizations } \\
$\psi$ & $\eta, \beta, \gamma$ & 500 & 1000 & 2500 & 5000 \\
\hline \multirow{3}{*}{0} & $3,3,1$ & 3.6 & 2.9 & 2.3 & 1.0 \\
& $3,2,1$ & 3.1 & 2.0 & 1.7 & 0.8 \\
& $3,1,1$ & 4.0 & 2.9 & 2.0 & 1.2 \\
$\pi / 6$ & $3,3,1$ & 4.6 & 3.4 & 2.1 & 1.1 \\
& $3,2,1$ & 4.2 & 2.4 & 1.5 & 1.1 \\
& $3,1,1$ & 4.9 & 3.7 & 2.3 & 1.7 \\
$\pi / 4$ & $3,3,1$ & 5.9 & 4.1 & 3.9 & 1.8 \\
& $3,2,1$ & 5.6 & 3.8 & 3.0 & 1.4 \\
& $3,1,1$ & 9.2 & 5.2 & 3.1 & 2.3 \\
$\pi / 3$ & $3,3,1$ & 11.0 & 7.8 & 7.4 & 4.2 \\
& $3,2,1$ & 10.3 & 6.5 & 4.3 & 3.4 \\
& $3,1,1$ & 14.6 & 10.8 & 9.4 & 7.7 \\
\hline \hline
\end{tabular}

\section{B. Fluctuation polarimetry-the case of known excitation field}

The two examples discussed here exemplify experimental situations where the incident field is unknown. Of course, the general expressions in Eq. (10) can be simplified and may also be used in situations where the experimental geometry allows for an alignment of the incident field to some known incident polarization. In this case, an ideal experimental geometry would consist of a linear excitation and a measurement performed along a codirection and a crossed direction of polarization. With a known linear incident state, Eq. (10) reduces to

$$
\begin{gathered}
M_{x}^{(1)}=\frac{4}{5} \delta_{1}+\bar{\alpha}^{2}, \\
M_{y}^{(1)}=\frac{3}{5} \delta_{1}, \\
M_{x x}^{(2)}=\frac{6}{35}\left(8 \delta_{1}^{2}+\frac{3}{7} \bar{\alpha}^{2} \delta_{1}-8 \bar{\alpha} \delta_{2}\right)+\bar{\alpha}^{4} .
\end{gathered}
$$

Notably, in this particular situation, it is possible to retrieve the morphological information about the scatterer without the need for correlated intensity distributions. Practically, this means that two independent intensity distributions can be recorded at different times or locations for a randomly oriented scatterer. This approach may be of interest in situations where the scattered intensity is very small, and the requirement to measure correlated polarimetric intensity distributions is a daunting task.

The above situation can be simplified even further when it is known a priori that the scatterer is rotationally symmetric. In this case, it is possible to obtain the shape aspect ratio by performing only a single measurement that is copolarized to the linear excitation in a manner similar to an earlier suggestion [7]. In this situation, the first and second moments of the detected distribution allow for such a quantification and the obtained expressions can be reduced from Eq. (10).

\section{Near-field scattering polarimetry}

Other applications of interest occur in the practice of nearfield optical microscopy. In this case, a sample is locally excited by either the field emitted through a tapered optical fiber with an aperture much smaller than the wavelength or by the field created around a sharp metallic tip placed in close proximity to the sample [8]. The polarization state of the excitation field is generally unknown but the procedure of analyzing fluctuations of the scattered intensity can also be used in this situation.

When the material system is optically inhomogeneous, its properties are described by the local polarizability. For a large class of materials where the inhomogeneities are on a scale much smaller than the radiation's wavelength, one can consider that this polarizability is constant in magnitude and orientation within some volume of interaction $V_{d}$ determined by the characteristics of the near-field probe (i.e., aperture size, tip sample separation, etc.). One can further assume that this effective polarizability only changes its orientation from 


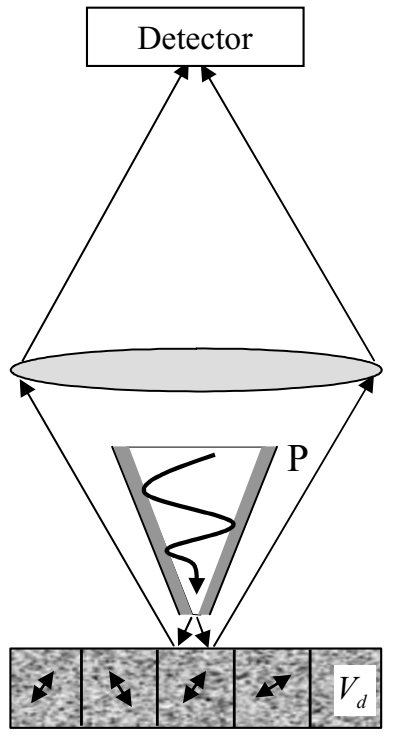

FIG. 2. The geometry of near-field scattering polarimetry. The probe of near-field microscope $P$ scans the heterogeneous sample having regions $V_{d}$ with uniformly oriented polarizability.

point to point and, during a scan, all possible orientations of the effective polarizability are realized (Fig. 2).

As opposed to the previous examples discussed, the field emerging from or surrounding the tip of a near-field scanning optical microscope (NSOM) is highly nonuniform. For a transmission aperture NSOM, for instance, a common approach is to approximate this with the field produced by diffraction from a small aperture in a perfectly conducting screen $[9,10]$. The interaction between this inhomogeneous electromagnetic field and a specific material system is complex and, in most cases, cannot be described analytically. The alternative is to use numerical techniques, such as the coupled dipole approximation (CDA), to calculate the scattering resulting from this interaction.

The coupled dipole approximation describes a continuum volume of a material as a finite array of polarizable point dipoles which react to both the local field and the complete interactions between all the other dipoles on the lattice $[11,12]$. The local dielectric properties of the medium are expressed by the polarizability of each individual dipole. For instance, local properties of homogenous materials, either optically isotropic or anisotropic, are being described by arrays of identical dipoles which all have the same magnitude and orientation.

To illustrate the procedure of stochastic polarimetry outlined before, we have used CDA to simulate the near-field scanning of a material consisting of an array of tightly packed anisotropic dipoles which are locally oriented in the same direction. The far-field scattered intensities were recorded in the backward direction, as in the conventional NSOM reflection mode shown in Fig. 2. The recorded farfield intensity ensembles were polarimetrically analyzed and the components of the polarizability tensor of the individual particles were calculated according to the method of stochastic polarimetry.

The results of anisotropic polarizability reconstruction in near-field geometry are shown in Fig. 3. Every data point

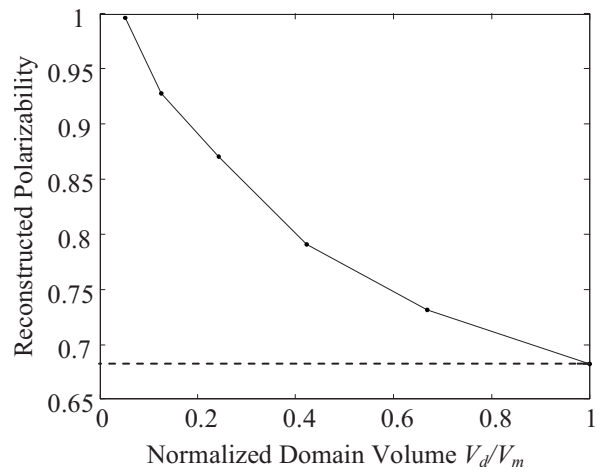

FIG. 3. Reconstructed polarizability (the $c$ component of diagonalized polarizability tensor normalized to the $a$ component) as a function of normalized domain volume $V_{d} / V_{m}$, where $V_{d}$ is the volume of uniform orientation of anisotropic polarizability and $V_{m}$ is the volume of interaction (solid line). The dashed line shows the exact value of polarizability. The parameters of CDA simulations are tip-sample separation $10 \mathrm{~nm}$, and modeling cube of 0.8 wavelength in size with $4 \times 10^{3}$ dipoles.

represents the result of averaging over 3000 realizations. As can be seen, the value of the calculated polarizability depends on the dimensions of the domain of uniform polarizability. Of course, the intrinsic values of the polarizability $(c / a=0.695)$ are recovered only when this domain volume is equal or larger than the volume of interaction. In practice, this volume of interaction depends on a number of factors including sample properties, tip characteristics, detection system, and the average intensity of excitation [13]. In the present simulation, the volume of interaction occupies almost the entire modeling volume of approximately $(0.8 \lambda)^{3}$, where $\lambda$ is the wavelength of incident field. Interestingly, our simulations indicate that this volume of interaction does not depend on the size of the tip's aperture (we tested apertures with sizes $25-50 \mathrm{~nm}$ ). This may happen because the volume of interaction has dimensions such that the field emerging from the tip appears to originate from a point dipole. Accordingly, the size of the tip's aperture effectively influences only the amplitude of this dipole field and does not change the overall field distribution. In the situation, where there is a significant averaging over the material structure within the volume of interaction, the resulting polarizability will correspond to some effective value depending on both the intrinsic material properties and structural morphology.

As a final observation we should note that the CDA simulations show that the reconstruction of the intrinsic polarizability of the anisotropic material modeled by individual dipoles is successful even when taking into account both the nonuniform excitation and the coupling between neighboring particles. This happens, because the probe illuminates only a highly localized volume. From the observation point in the wave zone, where polarimetric measurements are performed, this illuminated volume is seen as dipole excited by a field in a specific polarization state.

\section{CONCLUSIONS}

In this paper, we discussed in detail the general concept of stochastic scattering polarimetry [3]. We have demonstrated 
that the diagonal elements of the anisotropic polarizability tensor can be obtained by analyzing the statistical moments of polarimetrically measured intensity distributions. Most importantly, this can be done even in situations where the state of polarization of the incident field is unknown.

We presented and discussed numerical results pertaining to the specific cases where the orientation of the unknown polarizability was described by a uniform random distribution. Using the uniform distribution is not a conceptual limitation, as the general procedure of stochastic scattering polarimetry can also be applied to other distributions depending on the specifics of the experimental application. In our derivations we assumed linear polarimetric measurements in the wave zone. Analogous treatment can be elaborated in the case when a polarimetric detection system is based on circular polarization measurements. Also, it should be noted that when using this method, one obtains information not only about polarizability properties but also about properties of exciting field. As such, any known information about the material can also be used for probing the local properties of unknown electromagnetic fields [14].

We demonstrated that the method of stochastic scattering polarimetry can reconstruct the values of anisotropic polarizability both in the case of single nanoparticles excited by uniform field and in the case of interacting equivalent particles and the nonuniform excitation field that may occur in near-field measurements.

Finally, we have assumed in the examples presented that the particle or particles within the interaction volume are oriented in a single direction throughout each scattering event. However, the procedure of stochastic scattering polarimetry may be similarly used to determine properties of inhomogeneous materials, where the quantified polarizability corresponds to some effective property. This is apparent in the results presented in Fig. 3, where multiple domains of uniform anisotropy were coupled to determine the values of the far-field measurements. In the case of optically inhomogeneous media, the values of the effective polarizability will depend on the size of the highly localized excitation volume, a situation which is common in the practice of near-field microscopy, and will be the subject of future investigations.

\section{ACKNOWLEDGMENT}

This research was partially supported by the Air Force Office of Scientific Research.

\section{APPENDIX: EXPLICIT EXPRESSIONS FOR POLYNOMIALS $\boldsymbol{P}_{i j}^{(\boldsymbol{n})}$}

In this appendix we present the exact form of the polynomials $P_{i j}^{(n)}(\varepsilon)$ that enter the expressions for the second-order moments $M_{i j}^{(2)}$ in Eq. (10). In obtaining these results, an ensemble average was applied to the product of two intensities $I_{i} I_{j}$ and then terms containing different orders of $\delta_{1}$, and $\delta_{2}$ where collected.

$$
\begin{gathered}
P_{x x}^{(1)}(\varepsilon)=\frac{3}{35}\left\{\left(Q_{1}+3\right)^{2} \varepsilon^{2}+2\left[Q_{1} K+\left(Q_{2}+3\right)\right] \varepsilon+(K+3)\right\} \\
P_{x x}^{(2)}(\varepsilon)=\frac{6}{5}\left[Q_{1}\left(Q_{1}+3\right) \varepsilon^{2}+\left(2 Q_{1} K+Q_{2}\right) \varepsilon+K(K+3)\right] \\
P_{x x}^{(3)}(\varepsilon)=\frac{8}{35}\left[Q_{1}\left(Q_{1}-9\right) \varepsilon^{2}+\left(2 Q_{1} K-3 Q_{2}\right) \varepsilon+K(K-9)\right] \\
P_{x x}^{(4)}(\varepsilon)=Q_{1}^{2} \varepsilon^{2}+2 K Q_{1} \varepsilon+K^{2}, \\
P_{x y}^{(1)}(\varepsilon)=\frac{3}{35}\left[(L+3)^{2} \varepsilon^{2}+2(L+3) \varepsilon+9\right], \\
P_{x y}^{(2)}(\varepsilon)=\frac{3}{5}\left[\left(2 L Q_{1}+Q_{3}\right) \varepsilon^{2}+(1-L K) \varepsilon+K\right], \\
P_{y y}^{(2)}(\varepsilon)=\frac{6}{5}\left[L(L+3) \varepsilon^{2}+L \varepsilon\right], \\
P_{y y}^{(3)}(\varepsilon)=\frac{8}{35}\left[L(L-9) \varepsilon^{2}-3 L \varepsilon\right], \\
P_{y y}^{(4)}(\varepsilon)=L^{2} \varepsilon^{2}, \\
\left.\left.L(\varepsilon)=L Q_{1} \varepsilon^{2}+K L \varepsilon, Q_{3}+3\right) \varepsilon^{2}+2(7+10 L K) \varepsilon+3+K\right],
\end{gathered}
$$

where the following notations were used:

$$
\begin{gathered}
L=\cos ^{2}(\Phi), \quad K=\cos ^{2}(\psi), \\
Q_{1}=1-K-L+K L, \quad Q_{2}=1-L+K L, \quad Q_{3}=1-K+K L .
\end{gathered}
$$

The meaning of angles $\Phi$ and $\psi$ is that depicted in Fig. 1.
[1] M. Prummer, B. Sick, B. Hecht, and P. Urs Wild, J. Chem. Phys. 118, 9824 (2003).

[2] C. Sönnichsen and A. P. Alivisatos, Nano Lett. 5, 301 (2005).

[3] D. Haefner, S. Sukhov, and A. Dogariu, Phys. Rev. Lett. 100,
043901 (2008)

[4] L. Mandel and E. Wolf, Optical Coherence and Quantum Optics (Cambridge University Press, New York, 1995), Chap. 6.

[5] A. Stuart and J. Keith Ord, Kendall's Advanced Theory of Sta- 
tistics. Distribution Theory (John Wiley, New York, Toronto, 1994), Vol. 1.

[6] H. C. van de Hulst, Light Scattering by Small Particles (Dover, New York, 1981).

[7] E. Jakeman, Waves Random Media 5, 427 (1995).

[8] D. Courjon, Near-Field Microscopy and Near-Field Optics (Imperial College Press, London, 2003).

[9] C. J. Bouwkamp, Philips Res. Rep. 5, 321 (1950).

[10] R. D. Grober, T. Rutherford, and T. D. Harris, Appl. Opt. 35,
3488 (1996).

[11] E. M. Purcell and C. R. Pennypacker, Astrophys. J. 186, 705 (1973).

[12] B. T. Draine and P. J. Flatau, J. Opt. Soc. Am. A 11, 1491 (1994).

[13] A. Apostol, D. Haefner, and A. Dogariu, Phys. Rev. E 74, 066603 (2006).

[14] J. Ellis and A. Dogariu, Phys. Rev. Lett. 95, 203905 (2005). 